\title{
Positioning LBS to the third dimension
}

Edward Verbree, Sisi Zlatanova

Section GIS Technology, OTB Research Institute for Housing, Urban and Mobility Studies, Delft University of Technology

e.verbree@tudelft.nl, s.zlatanova@tudelft.nl

\section{Abstract}

Computer technology has evolved to a position of being able to handle large three-dimensional (3D) data sets. The third dimension is already taken for granted for visualisation on desktop machines. All GIS/CAD vendors are offering extended 3D tools for navigation and exploration of data. NASA World Wind and Google Earth demonstrate the possibilities of 3D to all users of the Web. The advances in geoDBMS are also striking; main stream DBMS support spatial data types, which can be adapted for handling of 3D data.

In the same way, mobile computing is experiencing a similar evolution. Large numbers of 3D computer games are already available for handhelds, which give new opportunities to geo-specialists of having 3D geoinformation on mobile devices. Despite the fact that 3D mobile hardware and software technologies are currently still behind desktop 3D in terms of capabilities, the expectations are for two or three times faster development of the mobile 3D market compared to its desktop counterpart.

Since location-based services (LBS) are among the first applications that naturally should consider the third dimension, we are going to investigate and evaluate the possible options for evolving to 3D LBS. The paper is going to concentrate and analyse on all the aspects of LBS: positioning, protocols, data retrieval and visualisation. Aspects typical for 3D data sets (large amounts of data, texturing, representations, data 
models) will be discussed in details. The paper will conclude with recommended topics for research and developments.

\section{Introduction}

Location-based Services are often referred to as utterly location-responsive Geographical Information Systems, with the aid of location-sensitive devices and location-aware services. Compared to mobile GIS, LBS is distinguished by considering the location of the mobile user in order to serve him or her with some added value information adaptive to that location. In this sense, these services are triggered to respond at certain locations according a specific user profile. As the user can be anywhere, the position fix should not be limited; here we will discuss LBS anytime, everywhere. The position determination should then be performed in full 3D space, thus not restricted only to outdoors and ground level applications.

In this chapter, we focus on the possibilities and limitations in 3D positioning with respect to LBS. After a short introduction to general available 3D positioning systems, the possibilities of combined GPSGalileo positioning in urban environments will be addressed by a simulation study on the availability of sufficient signal reception within city areas. This chapter concludes with a discussion on required research and developments.

\section{The need for 3D LBS anytime, everywhere}

Location-based Services are currently in use in many different areas and applications. The Location Services (OpenLS) of the Open Geospatial Consortium (OGC) comprise therefore an open platform for position access and location-based applications targeting mobile terminals. The OpenLS feature set is defined by the "Core Services and Abstract Data Types (ADT)". The most important aspect in these specifications is the 'GeoMobility Server', that provides requested information considering the position of the user. A Location Service Client sends a request for a position determination to the Gateway. The Gateway determines the position of the subscriber's mobile terminal and forwards this to the Location Service Client.

The minimum number of OpenLS services is currently defined as directory, route, location utility (geocoding and reverse geocoding), 
gateway and presentation (see Figure 1). These five core services are considered sufficient (Togt et.al., 2005) for a variety of use cases such as:

- Proximity: find something in a given area;

- Fencing: restrict the position of a user to within (our just outside) a given area;

- Navigation (compute route);

- Tracking ('record' the way of a user).

These services are, however, limited to $2 \mathrm{D}$, as the position ADT is restricted to latitude and longitude only.

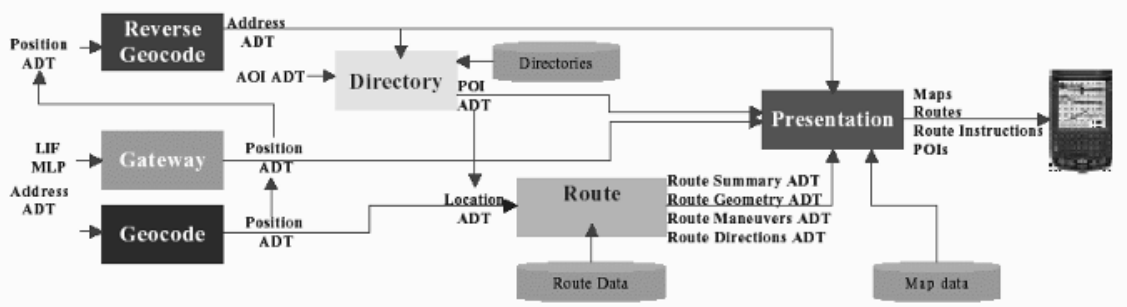

Fig. 1. GeoMobility Server (according to OpenLS)

3D LBS have to be able to ensure the same set of services, i.e., proximity, fencing, routing and tracking but in 3D (Zlatanova, et.al., 2005). Example of 3D requests would be:

- Proximity: 'show to me all the electrical switches for all floors in a building';

- Fencing: 'tell me when I am not at a dangerous floor of a building '

- Routing: 'compute a route from this floor to the ground level';

- Tracking: 'track this visitor all the way on his route from this floor to the ground level'.

In terms of core services as specified in OpenLS, 3D LBS needs to provide:

- 3D location utility service, i.e., 3D geo-coding and 3D reverse geocoding to transform a $3 \mathrm{D}$ position to a descriptive location and visaversa;

- 3D gateway service: to fetch a 3D position;

- 3D route service, i.e., give the route in multilevel constructions (buildings, viaducts, bridges, etc.); 
- 3D directory, access to an online directory to find the nearest or a specific 3D place, product or service;

- 3D presentation, i.e., 3D visualisation on mobile, hand-held devices and the appropriate interface for this

To be operational, these core services should have to work with a 3D position ADT, thus latitude, longitude, and height (related to some reference system). However, with respect to Location-based Services within the built environment, 3D positioning of location-sensitive devices anytime, everywhere, is still a challenge (Zlatanova et.al., 2003).

This issue is part of the developments of the OGC Web Services, phase 3 (OGC, 2006). Herein it is concluded that: "The present OpenLS services and information model are limited to outdoor navigation (i.e., the concepts of 'location' and 'navigation' are confined to outdoor activities.) An enhancement to the OpenLS services and information model is to support seamless indoor-outdoor navigation. The OpenLS services and information model will have to be modified to accommodate indoor location and navigation constructs". Therefore it is suggested to: "add a tracking service that supplies a position management and access capability and make first steps toward path-planning and navigation in buildings and other environments beyond the limits of road networks". By this kind of indooroutdoor navigation is should be made possible for "clients with mobile terminals to receive location and navigation guidance indoors or outdoors, as well as receive navigation guidance across indoor-outdoor and indoorindoor transition points (e.g., doorways)". Indoor location concepts must be supported for how people identify location for indoor environments, e.g., building, floor, room, etc. Indoor navigation concepts must also be supported for how people negotiate their way around indoor environments, e.g., 'park on level P1-P4', 'elevator to 3rd floor', 'right hall to room 310'.

It should be noticed, however, that the OpenLS specifications suggest a Gateway service that uses telecommunication networks for localization of the mobile user. Positioning with mobile communication networks is widely used for commercial, push LBS applications as it is quite easy to reach a group of cellular phone users within the area of a certain base station (Cell of Origin) and send them an advertisement SMS. It is also possible to position the users within a certain sector and range of the base station by Uplink Time Difference of Arrival. If that information is monitored over time and combined with a road network, the position of the cellular phone user can be detected in a more precise manner. For example, LogicaCMG has introduced the so-called Mobile Traffic Service where these locations are aggregated to real-time traffic information for the Dutch Province of Brabant (LogicaCMG, 2005). 
However, positioning with telecommunication networks based on just cell-id identification is inaccurate. Precise Mobile Network positioning requires considerable modifications to the current GSM network setup, or the use of next generation networks like UMTS. Due to the more or less planar arrangement of the GSM/UMTS beacons, accurate and reliable 3D positioning by mobile networks is not possible. Therefore, in the next sections we concentrate on other technologies for 3D positioning, focusing on their limitations.

\section{Limitations in 3D positioning technologies}

People can be positioned by a variety of other means such as Global Navigation Satellite Systems (GNSS) as GPS, location fingerprinting based on Wireless Local Area Network (WLAN), tags based on RFID, and other tracking approaches (Zlatanova et al, 2004). The location can also be given by using other non-coordinate related approaches such as address, floor, room, or a description of the environment. The location can be given (supposed the end-user posses the device/tool that would allow him to position himself) or requested (obtained by the system) by a user or by a control facility, for instance to locate a person in a building. Clearly, the localisation is one of the most important steps in providing any services. In this section, we will stress on the limitations of several 3D positioning approaches.

Theoretically, obtaining 3D coordinates at global scale is available. GPS devices, and other receivers to Global Navigation Satellite Systems (GNSS) like the in near future available Galileo can compute either Cartesian (X,Y,Z) or ellipsoidal (latitude, longitude, height) WGS84 coordinates. In multi-level 3D structures, the problem may come from two sides: geo-coding of the height and availability of sufficient satellites. The altitude is given as the distance to the WGS84 rotational ellipsoid. It is difficult to be linked to expressions used in daily life by references like 'on/under the bridge', 'floor', 'base', 'ceiling', 'top', etc. It is well known that a GNSS receiver cannot work inside or at other places with poor satellite coverage, i.e. less than four satellites in line-of-sight. Many systems exist that claim to solve that problem by applying another type of measurement technique. If it is not possible to detect enough GPS satellites in line-of-sight, some close-range pseudolites transmitters could be used also. For example, the company Noviriant offers the so-called Teralite XPS systems, a single frequency ground-based signal generator broadcasting XPS signals to mobile GPS+XPS receivers (Noviriant, 2006). 
For indoor applications, a more dedicated pseudolite-only setup could be used (Kee, 2001). However, if the user is free to move in height, the transmitters should be arranged in a $3 \mathrm{D}$ surround setting to obtain a reliable 3D position fix.

Assisted GPS (AGPS) combines the better of two worlds: GPS and Mobile Networks (Goran et al, 2001). When the Geo-Mobility Server requests a location, the wireless network sends the approximate location of the handset (generally the location of the closest cell site) to the location server. The location server then tells the handset which GPS satellites should be relevant for calculating its position. The handset takes a reading of the proper GPS signals, calculates its distance from all satellites in view and sends this information back to the location server to let its position be determined. In hard conditions like inside locations, it is still difficult or even impossible to 'see' enough satellites and thus to obtain a position fix. Moreover, inside conditions and urban canyons are known to have multipath problems, causing unreliable pseudo-ranges and thus fault determined positions.

In the last couple of years, location fingerprinting techniques using WLAN have been suggested for indoor areas where GPS does not work well (Keamarungsi et al, 2004, Xiang et al, 2004). Generally, the deployment of fingerprinting based positioning systems can be divided into two phases. First, in the offline phase, the location fingerprints are collected by performing a site-survey of the received signal strength (RSS) from multiple Access Points (APs). The RSS is measured with enough statistics to create a database or a table of predetermined RSS values. The vector of RSS values at a certain location is called the location fingerprint of that location. Second, in the online phase, a Mobile Station (MS) will report a sample measured vector of RSS from different APs to a central server. The server uses an algorithm, i.e. the Euclidian distance between the measured MSS vector and each fingerprint in the database, to estimate the location of the Mobile Station.

One major limitation of WLAN fingerprinting is, compared to GPS, the not controlled 'space segment', thus the locations and the signals strength of the WLAN Access Points. As these APs are not originated to be used as position beacons, they can be freely moved, rotated or in other ways disturbed from their original settings (i.e. switched off), causing a nonvalid location fingerprint. The estimation of the location in the online phase could be wrong, without any possibility to detect that. Although WLAN location fingerprinting could result in accurate results, they are not reliable. A second limitation has to be found in the mapping of the RSS values. For 3D location fingerprinting this mapping has to be performed in $3 \mathrm{D}$ space, thus sampling of fingerprints covering the $3 \mathrm{D}$ space. 


\section{Opportunities of combined GPS-Galileo positioning for urban environments}

Compared to other positioning sensors for LBS, for instance using radiosignals for mobile communication, a GNSS has the advantage of offering worldwide coverage. On the other hand, its weakest property is the requirement of, in principle, direct lines of sight to the transmitting satellites, which can be hard to realize particularly in urban environment, where typically most of the LBS applications will be used (Verbree et al, 2004).

One cannot always take measurements to determine whether a GNSS is available within urban areas. One has to realize that visibility of the satellites is not only determined by the location of the observer and obstructions around him, but also by the moment of observation as the satellites are in orbit. Besides, the actual observation of the availability of GPS during a day at or nearby a busy road crossing is impossible at all because of the traffic.

Simulation is the answer to these limitations. However, simulation requests a proper representation of the reality, both of the space segment as for the earth's surface. The actual orbits of the GPS are known by the almanac, but in comparing Galileo and GPS the nominal constellation of GPS is put side by side with the (proposed) orbits of Galileo. We have calculated the elevation and azimuth angles for the 24 GPS and the 27 Galileo satellites for each minute during a full daytime for the test area in Delft, at 52 degrees northern latitude in the Netherlands. At a fixed location on earth, the geometry of both GPS and Galileo repeat after approximate 24 hours.

The old city of Delft has very narrow streets with built-up areas of around 8-10 meters, with famous Dutch roof shapes. A partial area of the city is modeled in three dimensions by airborne laser-altimetry in combination with the cadastral data of the parcel boundaries. The 3D city model is built up by a polygonal representation of the canals, the streets and the roofs. The quaysides and the walls - the connections between the streets and the canals at one hand and the connection between streets and the roof tops at the other - are thought to be vertical and modeled as vertical polygons. The visibility calculation however is based on a Triangulated Irregular Network (TIN) that does not allow vertical polygon constrains. The solution to that problem is found in a buffering of both roofs and canals polygons by minus 10 centimeters. These datasets are the input for the surface model of this part of Delft represented by a TIN. A height-rendered image of this TIN is shown in figure 2. 


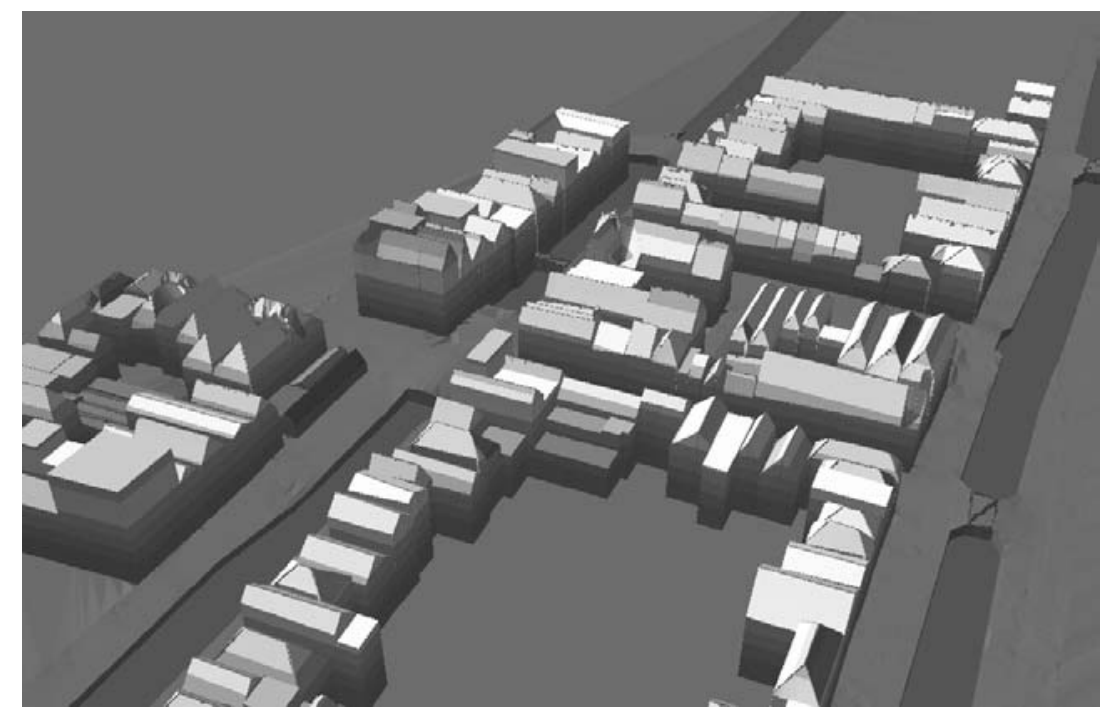

Fig. 2. 3D city model of Delft (TIN, height-rendered)

The actual visibility calculation is performed within the GIS package ArcView 3.2a with the extension 3D Analyst. The high-level scripting language Avenue allows fast prototyping of the algorithm with a proper visual feedback of the results within both $2 \mathrm{D}$ and $3 \mathrm{D}$ scenes. The simulation of the availability of GNSS consists of two algorithms. The first one calculates the total of targets (satellite positions at a certain time) seen from the observation point by:

Count $=0$

for each aTarget 'possible observable satellite

if (aTIN.LineOfSightsAsShapes (anObserver, aTarget,

ListOfShapes $)=$ True) then

Count $=$ Count +1

end

end

Satellite signal propagation is assumed to take place along geometric straight lines. The request aTIN.LineOfSightsAsShapes returns not only whether or not aTarget is visible from anObserver across aTIN surface, but also returns aListofshapes containing theobstacelePointz and the visible or invisible parts of the profile 
line. See figure 3 for the visual feedback of this calculation, with red the GPS visibility and within purple the Galileo visibility.

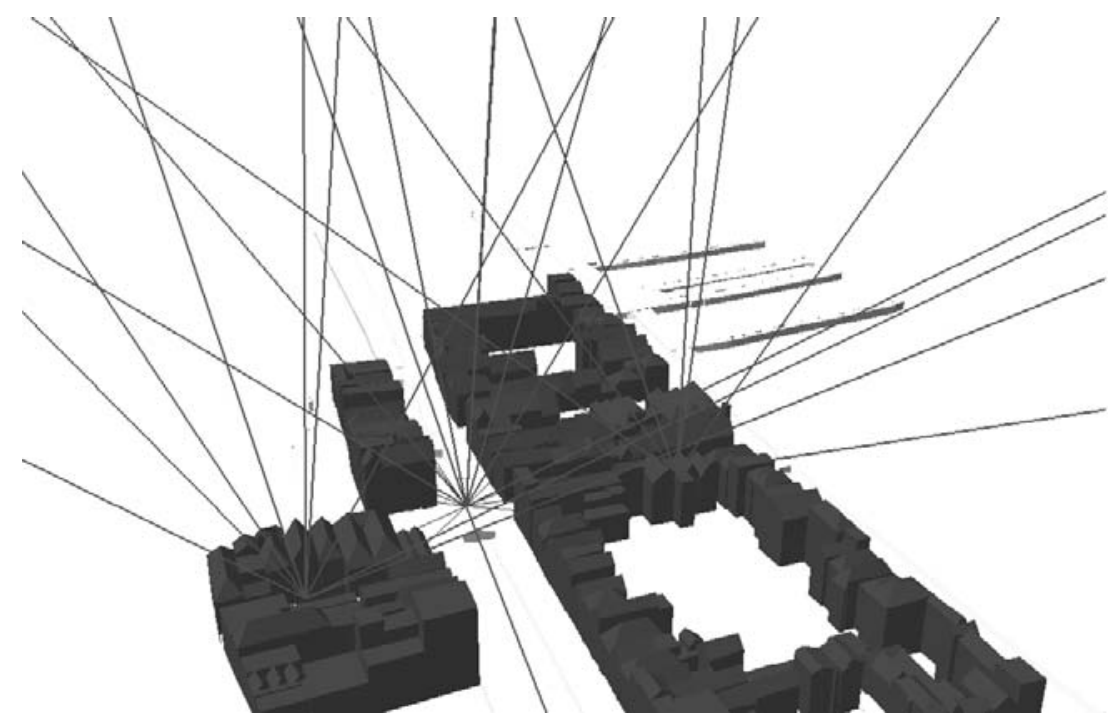

Fig. 3. Some examples of combined visibility of GPS and Galileo

The second algorithm calculates the availability of 'enough' satellites during a day time. We have chosen about 50 test observer points, with a height of $1.80 \mathrm{~m}$ above street level. For each of these observer points the total number of visible satellites during a day time is calculated. During a day time means $60 * 24=1440$ different constellations for both GPS and Galileo. Each target (24 GPS satellites and 27 Galileo satellites) within these constellations is checked by the request: aTIN. ObscuresTarget (anObserver, aTarget).

For GPS and Galileo alone observing four satellites simultaneously is the minimum for a position fix, without any preliminary knowledge as a known height. In combination of GPS and Galileo this requirement for sufficient availability holds true, but this demand can be extended with at least three GPS and two Galileo or two GPS and three Galileo satellites. Availability is analyzed here regardless the actual geometry of the visible satellites, which can have a large impact on the eventual position accuracy. The percentage of 'valid' cases gives an indication of the availability of GPS, Galileo and the combination of both within urban areas.

From this analysis, it can be concluded that the coverage of GPS alone is not sufficient within urban areas (i.e. less than $50 \%$ availability in narrow streets). However, for navigation purposes it has to be stated that 
the visibility on street crossings is far better than within street lanes. However, decisions where to go are made at crossings and car navigations systems use map-matching and auxiliary sensors to keep the car on track. A second consideration should be made upon the required visibility of four satellites. If the height is known and steady, (as in the streets) a position fix can be calculated out of the measurements to three visible satellites. This will improve the availability map considerably.

The calculated availability for Galileo alone is better than for GPS. The amount of proposed satellites (27 for Galileo compared to 24 for GPS) is due to this result. Again, the results will improve when relaxing the demand of four visible satellites to three.

The combination of GPS and Galileo is very promising, which is not surprisingly with 51 satellites to choose. Only half of them are above the horizon, but it is clearly shown that - besides very narrow streets - the availability of the combination is nearly $100 \%$. This result is however a little optimistic, because we have not taken into account the obstruction by trees and obstructions other than buildings. As a compromise, it is to be noted that for both Galileo and GPS the plain nominal satellite constellation was used.

As discussed in section 3, WLAN positioning is based on a kind of fingerprinting where providers have to generate first and foremost a database of signal characteristics of received radio waves at various, but known, locations. Once known, the position of the devices is determined by matching this database of digital fingerprints with the incoming signal. A basic form of fingerprinting could be applied to GNSS, as one of the main features of GNSS is the necessity of sufficient free line-of-sights to the satellites. As this characteristic can be determined within built environments in forehand, by considering a 3D city model, a GNSS fingerprint could be calculated. This GNSS fingerprint could increase the usability of GNSS within the built environment considerable.

\section{Discussion}

The general conclusion is that sufficient technological possibilities for 3D positioning exist but all of them do have their drawbacks with respect to Location-based Services, i.e., coverage (indoor/outdoor), availability (not anytime up and running), precision, reliability and integrity.

Most position systems are presented as 'stand-alone' solutions, due to commercial interests. As no system operates best under all circumstances, 
the reliability will be improved and ensured when the systems become more integrated.

The OpenLS specification has to be further extended to provide 3D core services:

- 3D location utility service, i.e. 3D geo-coding and 3D reverse geocoding to transform a 3D position to a descriptive location and visaversa;

- 3D gateway service: to fetch a 3D position;

- 3D route service, i.e. give the route in multilevel constructions (buildings, viaducts, bridges, etc.);

- 3D directory, access to an online directory to find the nearest or a specific 3D place, product or service;

- 3D presentation, i.e. 3D visualisation on mobile, hand-held devices and the appropriate interface for this.

In this respect, these core services should have to work with a 3D position ADT, thus latitude, longitude, and height (related to some reference system).

Presently, all 3D positioning techniques have particular limitations, but many developments are in progress and hopefully will take place in the coming few years. For example, 3D localisation based on a combined GPS and Galileo constellation - in conjunction with GNSS-fingerprinting - is expected to resolve some of the typical urban canyon problems, despite the performed calculation are a bit idealistic. When Galileo becomes operational, real tests should conclude on the effect of obstructing trees and other objects.

\section{References}

Goran M. Djuknic and Robert E. Richton, 2001, Geolocation and Assisted GPS, Computer, vol. 34, no. 2, pp. 123-125, February, 2001.

Kaemarungsi, Kamol and Prashant Krishnamurthy, 2004, Modeling of Indoor Positioning Systems Based on Location Fingerprinting, IEEE INFOCOM, 2004, $11 \mathrm{p}$.

Kee, Changdon, 2001, Centimeter-Accuracy Indoor Navigation Using GPS-Like Pseudolites, GPS World, November 2001.

LogicaCMG, 2006. www.logica-cmg.com; www.mts-live.com.

Noviriant, 2006. www.novariant.com/mining.

OGC, 2006. Open Geospatial Consortium: www.opengeospatial.org

Togt, R., E. Beinat, S. Zlatanova and H.J. Scholten, 2005, Location interoperability services for medical emergency operations during disasters, 
in: P.J.M. van Oosterom, S. Zlatanova \& E.M. Fendel (Eds.), Geoinformation for disaster management, Springer Verlag, Heidelberg, pp. 11271141.

Verbree, Edward, Christiaan Tiberius and George Vosselman, 2004, Combined GPS-Galileo positioning for Location Based Services in urban environment, in: Proceedings of the Symposium on Location Based Services \& Telecartography, 28-29 January, 2004, Vienna, Austria, pp 99-107.

Xiang, Z. et.al., 2004, A wireless LAN based positioning system, IBM Journal Res\&Dev, Vol 48, Sep./Nov., 2004, pp. 617-626.

Zlatanova, S. and E. Verbree, 2003, Technological Developments within 3D Location-based Services, International Symposium and Exhibition on Geoinformation 2003 (invited paper), 13-14 October 2003, Shah Alam, Malaysia, pp. 153-160.

Zlatanova, S. and E. Verbree, 2004, User tracking as an alternative positioning technique for LBS, in: Proceedings of the Symposium on Location Based Services \& Telecartography, 28-29 January, 2004, Vienna, Austria, pp. 109115.

Zlatanova, S. and E. Verbree, 2005, The third dimension in LBS: the steps to go, in: Proceedings of the 3rd Symposium on Location Based Services \& TeleCartography, 28-30 November 2005, Vienna, Austria, pp. 185-190. 